\title{
Comparable efficacy and less toxicity of pegylated liposomal doxorubicin versus epirubicin for neoadjuvant chemotherapy of breast cancer:
} a case-control study

This article was published in the following Dove Press journal: OncoTargets and Therapy

\section{Minjun Dong' \\ Liang Luo ${ }^{2}$ \\ Xiaogang Ying ${ }^{3}$ \\ Xianqiu Lu ${ }^{3}$ \\ Jianguo Shen' \\ Zhinong Jiang ${ }^{4}$ \\ Linbo Wang'}

'Department of Surgical Oncology,

${ }^{2}$ Department of Gastroenterology,

Sir Run Run Shaw Hospital, ${ }^{3}$ Zhejiang

University School of Medicine,

Hangzhou, People's Republic of

China; ${ }^{4}$ Department of Pathology,

Sir Run Run Shaw Hospital, Zhejiang

University School of Medicine,

Hangzhou, People's Republic of China
Background: Pegylated liposomal doxorubicin (PLD) and epirubicin are both superior variants of doxorubicin and are commonly applied as basic chemotherapeutics in breast cancer. However, the direct comparison of their efficacy and side effects has not been adequately reported. This study aimed to compare the efficacy and toxicity of PLD and epirubicin as neoadjuvant chemotherapy for invasive breast cancer.

Patients and methods: Women $(n=43)$ with invasive breast cancer who received neoadjuvant chemotherapy with the regimens containing PLD (PLD group) were analyzed and 1:2 matched with those $(\mathrm{n}=86)$ who received regimens containing epirubicin (epirubicin group) according to clinical TNM staging and taxane combination.

Results: The PLD group achieved similar clinical response rate in neoadjuvant chemotherapy compared to the epirubicin group (76.7\% vs $75.6 \%$ ). The PLD group had a lower rate of grade $3 \&$ 4 neutropenia (30.2\% vs $60.5 \%$ ), vomiting (7.0\% vs $28.0 \%$ ), and grade $3 \& 4$ alopecia $(9.3 \%$ vs $43.0 \%$ ), yet a higher rate of mouth ulceration (46.5\% vs $11.7 \%$ ). For the cardiac toxicity, the PLD group had a significantly lower rate of ventricular premature beat compared with the epirubicin group ( $7.0 \%$ vs $20.9 \%, p=0.043$ ), and cardiac ultrasonography monitoring showed non-significantly less PLD group patients' left ventricular ejection fraction decline more than $10 \%$ compared with the epirubicin group $(4.7 \%$ vs $8.1 \%, p=0.463)$.

Conclusion: In neoadjuvant chemotherapy for invasive breast cancer, PLD provides potentially similar efficacy and relatively less toxicity compared to epirubicin.

Keywords: neoadjuvant chemotherapy, pegylated liposomal doxorubicin, epirubicin, breast cancer, efficacy

\section{Introduction}

Breast cancer, the most common cancer diagnosed in women and the second most common cancer overall, has been a major threat to global public health. ${ }^{1,2}$ Chemotherapy drugs and regimens for breast cancer have been improving during the past halfcentury, and have distinctly improved the outcome of breast cancer patients. Further, as the neoadjuvant chemotherapy has been widely used, more breast cancer patients get the chance to accept the breast conserving surgery, and more patients who are initially inoperable can be down staged as operable. However, breast cancer patients benefiting from the use of more powerful chemotherapy agents has resulted in a proportionate increase in toxicities. Anthracycline-based neoadjuvant chemotherapy is the recommended therapy for early-stage and local advanced breast cancer. However, this therapy
Correspondence: Linbo Wang Department of Surgical Oncology, Sir Run Run Shaw Hospital, Zhejiang University School of Medicine, 3 East Qingchun Road, Hangzhou 310016 , People's Republic of China Fax +86 57I 860448I7 Email linbowang@zju.edu.cn 
can provoke a toxic host response, such as nausea, vomiting, and leucopenia that sometimes makes treatment unfeasible. ${ }^{3}$ In addition, elderly patients and those who receive multiple courses of anthracycline-based chemotherapy are at risk for cardiac toxicity, which can lead to heart failure. ${ }^{4,5}$

Pegylated liposomal doxorubicin (PLD) has been demonstrated to achieve similar efficacy to conventional doxorubicin, while it reduces the toxicity profile, with a lower incidence of nausea/vomiting and cardiotoxicity in metastatic breast cancer. ${ }^{6,7}$ Epirubicin is another commonly used anthracycline in breast cancer. The aim of the current study was to compare the efficacy and toxicity profile of PLD and epirubicin as neoadjuvant chemotherapy for stage II-III invasive breast cancer.

\section{Patients and methods Study design}

Approval to utilize the breast cancer database of the authors' institutions was obtained from the medical ethics committee of Sir Run Run Shaw Hospital. Informed consent was waived by the ethics committee for this retrospective study which did not involve intervention with the patients, and all data was kept confidential. All protocols were in accordance with the Declaration of Helsinki. Approval to utilize the authors' institutions' breast cancer database was obtained from the sample size calculation and showed that at least 25 pairs were needed for examining the difference of efficacy, and at least 39 pairs were needed for examining the difference of the side effects for this study. From June 2012 to December 2015, 43 patients with stage II to III breast cancer underwent neoadjuvant chemotherapy with PLDbased regimens at the Department of Surgical Oncology, Sir Run Run Shaw Hospital, Zhejiang University School of Medicine (Hangzhou, People's Republic of China). For each of the 43 patients, two control breast cancer patients who used epirubicin-based regimens as neoadjuvant chemotherapy were matched according to the clinical staging and the combination of taxanes. Therefore, a total of 129 patients were enrolled in the present case-control study.

Patients received combined regimens either with PLD [35 mg/m $\left.\mathrm{m}^{2}\right]$ or epirubicin [100 $\left.\mathrm{mg} / \mathrm{m}^{2}\right]$ intravenous (iv) infusion for up to 60 min every 3 weeks. The chemotherapy regimen and intensity were chosen according to the St Gallen recurrence risk assessment. ${ }^{8}$ As per protocol, dose modifications of either of the two drugs was permitted for palmarplantar erythrodysesthesia, hematological toxicity and other grade 3 and 4 adverse events. Neoadjuvant chemotherapy was to be discontinued when the tumor size shrank to reach the goal of performing breast conserving surgery or radical mastectomy. Post-operation treatment strategy was made according to the National Comprehensive Cancer Network guideline for breast cancer, such as completing the residual chemotherapy, radiotherapy for breast conserving patients and lymph node metastasis patients, herceptin therapy for HER2-positive patients and endocrine therapy for estrogen receptor/progesterone receptor (ER/PR)-positive patients.

\section{Clinical assessments}

Every patient underwent ultrasound and a mammography for the local disease assessment, and breast MRI was arranged for those candidates of breast conserving surgery. Core needle biopsy on every breast tumor and fine needle biopsy on enlarged lymph node were performed. Pathologic assessment included tumor histology and ER/PR/HER2/Ki67 IHC staining. ER/PR-positive was defined as $\geq 1 \%$ expression, HER2-positive was defined as IHC 3+ or FISH test amplified, Ki67-positive was defined as $>14 \% .{ }^{9}$ Tumor size and regional lymph nodes were evaluated every two chemo cycles with ultrasound for each patient, and every 3-4 chemo cycles with MRI for patients who planned to access breast conserving surgery. Clinical tumor response was evaluated using the Response Evaluation Criteria in Solid Tumors Criteria version $1.0^{10,11}$ and pathological judgment, pathological complete response (pCR) was defined as those who had noninvasive or in situ cancer in the breast specimen as well as those in whom no residual cancer was identified, regardless of the status of the axillary or regional lymph node. ${ }^{12}$

During the treatment, assessment of physical signs, performance status, adverse events, complete blood count, and serum chemistry were managed weekly or more frequently when in need. Cardiac ultrasonography was carried out before the neoadjuvant chemotherapy started and reinspected when the patient reported heart-associated symptoms or every 2-3 chemo cycles and continued after the whole cycles of chemotherapy every 3 months at least for half a year. All side effects were assessed by nurses according to World Health Organization (WHO) and the American Society of Clinical Oncology standards.

\section{Statistical analysis}

Chi-square tests were used to examine the base-line characteristics of the two groups, including: Eastern Cooperative Oncology Group score, menstrual states, ER/HER2/Ki67 status, molecular subtype, clinical T staging, and histology. Toxicities and tumor response were also analyzed with Chi-square tests. One-way ANOVA was used to compare the chemo cycles before surgery. Treatment-related toxicities were reported and summarized by the highest grade per patient. All statistical tests were two-sided and a $p$-value of 
less than 0.05 was considered statistically significant. Statistical analysis was performed using SPSS 19 for Windows ${ }^{\mathbb{R}}$ (IBM Corporation, Armonk, NY, USA).

\section{Results}

\section{Patient characteristics}

The PLD group and the epirubicin group were comparable in respect to demographic and disease characteristics, including age, Eastern Cooperative Oncology Group score, menstrual states, ER/HER2/Ki67 status, molecular subtype, clinical T staging, chemo cycles before surgery, and histology (Table 1).

Table I Baseline characteristics

\begin{tabular}{|c|c|c|c|}
\hline Index & $\begin{array}{l}\text { PLD } \\
(n=43)\end{array}$ & $\begin{array}{l}\text { Epirubicin } \\
(n=86)\end{array}$ & $p$-value \\
\hline Age (years) & $52.1 \pm 8.6$ & $50.4 \pm 8.2$ & 0.262 \\
\hline ECOG score & & & 0.593 \\
\hline 0 & $42(97.7 \%)$ & $73(96.5 \%)$ & \\
\hline I & I $(2.3 \%)$ & $3(3.5 \%)$ & \\
\hline Menstrual states & & & 0.108 \\
\hline Premenopause & $12(25.5 \%)$ & 35 (37.8\%) & \\
\hline Postmenopause & $3 \mathrm{I}(73.8 \%)$ & $5 \mathrm{I}(62.2 \%)$ & \\
\hline ER status & & & 0.426 \\
\hline Positive ( $\geq 1 \%$ ) & 27 (62.8\%) & $5 \mathrm{I}(59.3 \%)$ & \\
\hline Negative (<1\%) & $16(37.2 \%)$ & $35(40.7 \%)$ & \\
\hline HER2 status & & & 0.414 \\
\hline Positive & $13(30.2 \%)$ & $23(26.7 \%)$ & \\
\hline Negative & $30(69.8 \%)$ & $63(73.3 \%)$ & \\
\hline Ki67 & & & 0.426 \\
\hline Low $(\leq 14 \%)$ & 15 (34.9\%) & $33(38.4 \%)$ & \\
\hline High (>I4\%) & $28(65.1 \%)$ & $53(61.6 \%)$ & \\
\hline Molecular subtypes & & & 0.904 \\
\hline Luminal A & II (25.6\%) & $26(30.2 \%)$ & \\
\hline Luminal B & $15(34.9 \%)$ & $25(29.1 \%)$ & \\
\hline HER2 enriched & $4(9.3 \%)$ & $9(10.5 \%)$ & \\
\hline Triple negative & $13(30.2 \%)$ & $26(30.2 \%)$ & \\
\hline Clinical T staging & & & 0.991 \\
\hline 1 & $2(4.7 \%)$ & $5(5.8 \%)$ & \\
\hline 2 & $32(74.4 \%)$ & $64(74.4 \%)$ & \\
\hline 3 & $5(11.6 \%)$ & $9(10.5 \%)$ & \\
\hline 4 & $4(9.3 \%)$ & $8(9.3 \%)$ & \\
\hline Histology & & & 0.852 \\
\hline Invasive ductal carcinoma & 40 (93.0\%) & $80(93.0 \%)$ & \\
\hline Invasive lobular carcinoma & I (2.3\%) & $\mathrm{I}(\mathrm{I} .2 \%)$ & \\
\hline Others & $2(4.7 \%)$ & $5(5.8 \%)$ & \\
\hline Chemo cycles before surgery & $4.9 \pm 2.3$ & $4.7 \pm 2.3$ & 0.679 \\
\hline Chemo regimen before surgery & & & Paired \\
\hline With taxanes & 28 & 56 & \\
\hline Without taxanes & 15 & 30 & \\
\hline Clinical TNM staging & & & Paired \\
\hline Ila & 16 & 32 & \\
\hline Ilb & 9 & 18 & \\
\hline Illa & 14 & 28 & \\
\hline IIllb & 4 & 8 & \\
\hline
\end{tabular}

Abbreviations: ECOG, Eastern Cooperative Oncology Group; ER, estrogenreceptor; HER2, human epidermal growth factor receptor 2; PLD, Pegylated liposomal doxorubicin.
Table 2 Clinical effect of neoadjuvant chemotherapy

\begin{tabular}{|c|c|c|c|}
\hline & $\begin{array}{l}\text { PLD } \\
(n=43)\end{array}$ & $\begin{array}{l}\text { Epirubicin } \\
(n=86)\end{array}$ & $\overline{p \text {-value }}$ \\
\hline Response to chemotherapy & & & 0.991 \\
\hline PR & $24(55.8 \%)$ & 49 (57.0\%) & \\
\hline CR & $9(20.9 \%)$ & $16(18.6 \%)$ & \\
\hline SD & 9 (20.9\%) & 19 (22.1\%) & \\
\hline PD & I $(2.3 \%)$ & $2(2.3 \%)$ & \\
\hline Response rate $(P R+C R)$ & $33(76.7 \%)$ & 65 (75.6\%) & 0.884 \\
\hline
\end{tabular}

Abbreviations: PLD, pegylated liposomal doxorubicin; CR, complete response; $\mathrm{PR}$, partial response; PD, progressive disease; SD, stable disease.

\section{Efficacy}

The two groups achieved comparable clinical responses to neoadjuvant chemotherapy, the difference of the rate of partial response (PR), complete response (CR), stable disease (SD), and progressive disease (PD), between the two groups were all non-significant, respectively. The difference of general response rate (combined PR and CR) for the two groups was also non-significant (Table 2).

Pathological review of the response to neoadjuvant chemotherapy found no significant difference between the PLD group and epirubicin group in pCR rate $(16.3 \%$ vs $11.6 \%$, $p=0.317$ ) (Figure 1).

Breast conserving rate in the PLD group and the epirubicin group was $39.5 \%$ and $11.6 \%$, respectively. There was one patient in each group who finally received palliative surgery. The rest went under modified radical mastectomy (Table 3).

\section{Noncardiac toxicity}

According to the recorded treatment-related noncardiac toxicity, these two groups' patients experienced different types and different levels of toxicity. Comparing with the epirubicin group, the PLD group had a lower rate of grade 3 neutropenia, vomiting, less grade 3 alopecia, but a higher rate

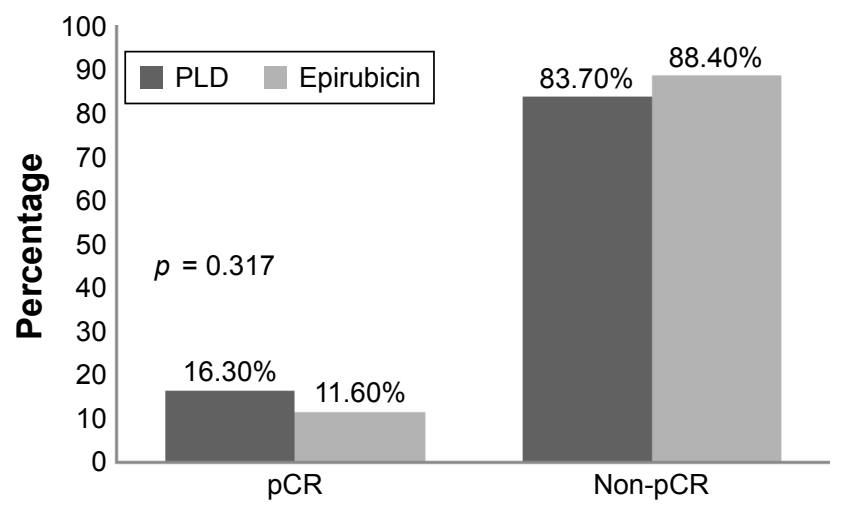

Figure I Pathological response to neoadjuvant chemotherapy. Abbreviations: PLD, pegylated liposomal doxorubicin; PCR, pathological complete response. 
Table 3 Operation type

\begin{tabular}{lll}
\hline Surgery type & $\begin{array}{l}\text { PLD } \\
(\mathbf{n}=\mathbf{4 3})\end{array}$ & $\begin{array}{l}\text { Epirubicin } \\
(\mathbf{n}=\mathbf{8 6})\end{array}$ \\
\hline Breast conserving surgery & $17(39.5 \%)$ & $10(11.6 \%)$ \\
Modified radical mastectomy & $25(58.1 \%)$ & $75(87.2 \%)$ \\
Palliative surgery & $\mathrm{I}(2.3 \%)$ & $1(1.2 \%)$ \\
\hline
\end{tabular}

Abbreviation: PLD, pegylated liposomal doxorubicin.

of mouth ulceration. In addition, the two groups had similar rate of anemia, mucositis, and palmar-plantar erythrodysesthesia (Table 4). (The PLD group had two patients' data missing and the epirubicin group had three patients' data missing).

\section{Cardiac toxicity}

The surveillance on ECG suggested that the PLD group patients reported a significantly lower rate of ventricular premature beat compared with the epirubicin group. The left ventricular ejection fractions (LVEF) decline of more than $10 \%$ were non-significantly less in the PLD group than in the epirubicin group during the short follow-up period (Table 5).

\section{Discussion}

PLD and epirubicin are both superior variants of doxorubicin and are commonly applied as basic chemotherapeutics in breast cancer. However, the direct comparison of their efficacy and side effects has not been adequately reported.

Epirubicin has been widely used in the neoadjuvant and adjuvant chemotherapy for invasive breast cancers, with many large clinical trials recommending it as a fundamental chemotherapeutic. ${ }^{13-16}$ However, some clinical trials have also pointed out that the use of epirubicin as well as doxorubicin have been partially restricted because of the increased fatigue, neutropenia, and thrombocytopenia. ${ }^{17,18}$

PLD has offered an alternative to doxorubicin for women with metastatic breast cancer. In a large randomized Phase III trial of PLD versus doxorubicin in first-line treatment of women with metastatic breast cancer, PLD and doxorubicin were broadly comparable in efficacy, but PLD had a different safety profile, with significantly reduced cardiac toxicity as compared with doxorubicin. ${ }^{7}$ Because of the obvious cardiac toxicity of doxorubicin-based chemo regimens, our center had reduced the use of doxorubicin and has used epirubicin more often as an alternative for years. So far as we know, there has not been any published study directly comparing both the efficacy and safety of PLD and epirubicin.

The use of PLD-based neoadjuvant chemotherapy regimen is in the growing, a few studies had reported their experience of the use of PLD. In 2002, the Phase II study by the Hellenic Cooperative Oncology Group was the first to point out that PLD was active in breast neoadjuvant chemotherapy and its toxicity was manageable. ${ }^{19}$ Then, Manguso et al reported satisfactory efficacy and low rate of cardiac, liver, hematological, and other toxicity while using neoadjuvant chemotherapy with low dose of PLD plus weekly paclitaxel in operable and locally advanced breast cancer. ${ }^{20}$ Our clinical practice was consistent with the previous studies and other trials..$^{21,22}$

Neoadjuvant chemotherapy for local advanced breast cancer is an important strategy for patients in order to get a better chance of breast conserving surgery ${ }^{23}$ or turn the initially inoperable disease into operable. $.^{24} \mathrm{PR}, \mathrm{CR}$, and pCR are good responses to neoadjuvant chemotherapy, and the response rate is comparable between the PLD group and the epirubicin group, which provides a more apparent evidence of the efficacy of PLD.

The $\mathrm{pCR}$ rate is not the major point to compare the efficacy, while the response rate is. Though the pCR rate differed between the two groups, the difference was not significant. We analyzed our raw data and found that in the same T2 staging, the average tumor size of the PLD group was non-significantly smaller than the epirubicin group $(3.42 \pm 0.64 \mathrm{~cm}$ vs $3.84 \pm 0.91 \mathrm{~cm})$, and this may have contributed to the difference of the $\mathrm{pCR}$ rate.

Table 4 Treatment-related noncardiac toxicity

\begin{tabular}{|c|c|c|c|c|c|c|c|}
\hline \multirow[t]{2}{*}{ Adverse events } & \multicolumn{3}{|c|}{ PLD $(n=4 I)$} & \multicolumn{3}{|c|}{ Epirubicin $(n=83)$} & \multirow[t]{2}{*}{$p$-value } \\
\hline & Grade I-2 & Grade 3 & Grade 4 & Grade I-2 & Grade 3 & Grade 4 & \\
\hline Neutropenia & $29(67.4 \%)$ & $13(30.2 \%)$ & 0 & $34(39.5 \%)$ & $49(57.0 \%)$ & $3(3.5 \%)$ & $0.006 *$ \\
\hline Anemia & $12(27.9 \%)$ & $2(4.7 \%)$ & 0 & $29(33.7 \%)$ & $8(9.3 \%)$ & 0 & 0.444 \\
\hline Vomiting & $3(7.0 \%)$ & 0 & 0 & $20(23.3 \%)$ & $4(4.7 \%)$ & 0 & $0.020 *$ \\
\hline Mucositis & $12(27.9 \%)$ & $3(7.0 \%)$ & 0 & I8 (20.9\%) & $2(2.3 \%)$ & 0 & 0.255 \\
\hline Mouth ulceration & 15 (34.9\%) & $5(11.6 \%)$ & 0 & $6(7.0 \%)$ & $4(4.7 \%)$ & 0 & $<0.00 I^{*}$ \\
\hline Alopecia & $27(62.8 \%)$ & $4(9.3 \%)$ & 0 & $49(57.0 \%)$ & $32(37.2 \%)$ & $5(5.8 \%)$ & $<0.00 I^{*}$ \\
\hline Palmar-plantar erythrodysesthesia & $7(16.3 \%)$ & $4(9.3 \%)$ & $2(4.7 \%)$ & $8(9.3 \%)$ & $5(5.8 \%)$ & 0 & 0.089 \\
\hline
\end{tabular}

Note: $*_{p}<0.05$.

Abbreviation: PLD, pegylated liposomal doxorubicin. 
Table 5 Treatment-related cardiac toxicity

\begin{tabular}{llll}
\hline Adverse events & PLD & Epirubicin & p-value \\
\hline Ventricular premature beat & $3(7.0 \%)$ & $18(20.9 \%)$ & $0.043^{*}$ \\
LVEF decline $>10 \%$ & $2(4.7 \%)$ & $7(8.1 \%)$ & 0.463 \\
\hline
\end{tabular}

Note: $*_{p}<0.05$.

Abbreviations: PLD, pegylated liposomal doxorubicin; LVEF, left ventricular ejection fraction.

The reason why the conservative surgery rate was quite different is complicated, including the education background, financial resources, acceptability of radiotherapy, and so on. In our study, we estimated that about $50 \%$ of both group patients could have a chance to receive the conservative breast surgery; among them, it seems obvious that more rich patients chose PLD with the hope of less side effects of chemotherapy, and these patients had higher demand of their appearance and life quality.

Patients treated with PLD experienced less myelosuppression, vomiting, and alopecia than those treated with epirubicin, even though there was greater use of supportive care including 5-HT3 antagonists and granulocyte colonystimulating factor among patients treated with epirubicin. However, the PLD group patients reported higher rate of mouth ulceration, which induced some days of pain in the mouth, and impacted their appetite. The two groups had similar rates of anemia, mucositis and palmar-plantar erythrodysesthesia.

Epirubicin demonstrated less cardiac toxicity than doxorubicin on an equimolar basis, ${ }^{25}$ and PLD also showed reduced cardiac toxicity as compared with doxorubicin. ${ }^{7}$

According to the American Society of Clinical Oncology cancer care system, arrhythmia or an irregular heartbeat belong to the heart toxicity, but the specific arrhythmia types were not described. In our daily work, we arranged ECG for every patient before each cycle of chemotherapy and recorded the symptoms. The ventricular premature beats seemed to happen more frequently and patients complained more about this discomfort.

During the short term of neoadjuvant chemotherapy and in the following 6 months, there were significantly less ventricular premature beats and a similar drop of LVEF in the PLD group compared with the epirubicin group, although the dexrazoxane was selectively used in the epirubicin group for the relatively high-risk patients according to the European Society for Medical Oncology clinical practice guideline recommendation. ${ }^{22}$

In conclusion, although this retrospective study was less persuasive than the prospective ones, we designed this matched case-control study to increase the power of the evidence. For patients with stage II-III invasive breast cancer who are to receive neoadjuvant chemotherapy, PLD has similar efficacy and less toxicity compared with epirubicin. Thus, PLD may be an important new therapeutic option for breast cancer patients, especially for the patients who are at increased cardiac risk (the elderly, patients with specific cardiac risk factors, and patients who have been previously treated with anthracyclines). Based on this study, we have started recruiting patients in a prospective randomized controlled trial which will be analyzed and reported in a few years.

\section{Acknowledgments}

This investigation was supported in part by the specialized scientific research fund projects of Zhejiang Provincial Population and Family Planning Commission, grant No 2017181152; and supported in part by Youth Talent Project of Zhejiang Provincial Population and Family Planning Commission, grant No 2018254316.

\section{Disclosure}

The authors report no conflicts of interest in this work.

\section{References}

1. Siegel RL, Miller KD, Jemal A. Cancer statistics, 2016. CA Cancer J Clin. 2016;66(1):7-30.

2. Chen W, Zheng R, Baade PD, et al. Cancer statistics in China, 2015. CA Cancer J Clin. 2016;66(2):115-132.

3. Ryberg M, Nielsen D, Cortese G, Nielsen G, Skovsgaard T, Andersen PK. New insight into epirubicin cardiac toxicity: competing risks analysis of 1097 breast cancer patients. J Natl Cancer Inst. 2008;100(15): $1058-1067$.

4. Gil-Gil MJ, Bellet M, Morales S, et al. Pegylated liposomal doxorubicin plus cyclophosphamide followed by paclitaxel as primary chemotherapy in elderly or cardiotoxicity-prone patients with high-risk breast cancer: results of the phase II CAPRICE study. Breast Cancer Res Treat. 2015;151(3):597-606.

5. Jensen BV. Cardiotoxic consequences of anthracycline-containing therapy in patients with breast cancer. Semin Oncol. 2006;33(3 Suppl. 8): S15-S21.

6. Gabizon A, Isacson R, Rosengarten O, Tzemach D, Shmeeda H, Sapir R. An open-label study to evaluate dose and cycle dependence of the pharmacokinetics of pegylated liposomal doxorubicin. Cancer Chemother Pharmacol. 2008;61(4):695-702.

7. O'Brien ME, Wigler N, Inbar M, et al. Reduced cardiotoxicity and comparable efficacy in a phase III trial of pegylated liposomal doxorubicin $\mathrm{HCl}$ (CAELYX/Doxil) versus conventional doxorubicin for first-line treatment of metastatic breast cancer. Ann Oncol. 2004;15(3):440-449.

8. Bauer K, Parise C, Caggiano V. Use of ER/PR/HER2 subtypes in conjunction with the 2007 St Gallen Consensus Statement for early breast cancer. BMC Cancer. 2010;10:228.

9. Munzone E, Botteri E, Sandri MT, et al. Prognostic value of circulating tumor cells according to immunohistochemically defined molecular subtypes in advanced breast cancer. Clin Breast Cancer. 2012;12(5): 340-346.

10. Khokher S, Qureshi MU, Chaudhry NA. Comparison of WHO and RECIST criteria for evaluation of clinical response to chemotherapy in patients with advanced breast cancer. Asian Pac J Cancer Prev. 2012; 13(7):3213-3218. 
11. Nishino M, Jagannathan JP, Ramaiya NH, Van den Abbeele AD. Revised RECIST guideline version 1.1: What oncologists want to know and what radiologists need to know. AJR. 2010;195(2):281-289.

12. Bear HD, Anderson S, Brown A, et al. The effect on tumor response of adding sequential preoperative docetaxel to preoperative doxorubicin and cyclophosphamide: preliminary results from national surgical adjuvant breast and bowel project protocol B-27. J Clin Oncol. 2003;21(22):4165-4174.

13. Martin M, Rodríguez-Lescure A, Ruiz A, et al. Randomized phase 3 trial of fluorouracil, epirubicin, and cyclophosphamide alone or followed by Paclitaxel for early breast cancer. J Natl Cancer Inst. 2008;100(11): 805-814.

14. French Adjuvant Study Group. Benefit of a high-dose epirubicin regimen in adjuvant chemotherapy for node-positive breast cancer patients with poor prognostic factors: 5-year follow-up results of French Adjuvant Study Group 05 randomized trial. J Clin Oncol. 2001;19(3):602-611.

15. Levine MN, Pritchard KI, Bramwell VH, et al. Randomized trial comparing cyclophosphamide, epirubicin, and fluorouracil with cyclophosphamide, methotrexate, and fluorouracil in premenopausal women with node-positive breast cancer: update of National Cancer Institute of Canada Clinical Trials Group Trial MA5. J Clin Oncol. 2005; 23(22):5166-5170.

16. Roché H, Fumoleau P, Spielmann M, et al. Sequential adjuvant epirubicin-based and docetaxel chemotherapy for node-positive breast cancer patients: the FNCLCC PACS 01 Trial. J Clin Oncol. 2006; 24(36):5664-5671.

17. Schwentner L, Harbeck N, Singer S, et al. Short term quality of life with epirubicin-fluorouracil-cyclophosphamid (FEC) and sequential epirubicin/cyclophosphamid-docetaxel (EC-DOC) chemotherapy in patients with primary breast cancer - results from the prospective multi-center randomized ADEBAR trial. Breast. 2016;27:69-77.
18. Earl HM, Hiller L, Dunn JA, et al. NEAT: National Epirubicin Adjuvant Trial - toxicity, delivered dose intensity and quality of life. Br J Cancer. 2008;99(8):1226-1231.

19. Golshan M, Cirrincione CT, Sikov WM, et al. Impact of neoadjuvant chemotherapy in stage II-III triple negative breast cancer on eligibility for breast-conserving surgery and breast conservation rates: surgical results from CALGB 40603 (Alliance). Ann Surg. 2015;262(3): 434-439; discussion 438-439.

20. Manguso N, Gangi A, Giuliano AE. Neoadjuvant chemotherapy and surgical management of the axilla in breast cancer: a review of current data. Oncology (Williston Park). 2015;29(10):733-738.

21. Torti FM, Bristow MM, Lum BL, et al. Cardiotoxicity of epirubicin and doxorubicin: assessment by endomyocardial biopsy. Cancer Res. 1986;46(7):3722-3727.

22. Bovelli D, Plataniotis G, Roila F. Cardiotoxicity of chemotherapeutic agents and radiotherapy-related heart disease: ESMO Clinical Practice Guidelines. Ann Oncol. 2010;21(Suppl. 5):v277-v282.

23. Mathew J, Asgeirsson KS, Cheung KL, Chan S, Dahda A, Robertson JF. Neoadjuvant chemotherapy for locally advanced breast cancer: a review of the literature and future directions. Eur J Surg Oncol. 2009;35(2): $113-122$.

24. Alassas M, Chu Q, Burton G, Ampil F, Mizell J, Li BD. Neoadjuvant chemotherapy in stage III breast cancer. Am Surg. 2005;71(6):487-492.

25. Robert J. Preclinical assessment of anthracycline cardiotoxicity in laboratory animals: predictiveness and pitfalls. Cell Biol Toxicol. 2007; 23(1):27-37.
OncoTargets and Therapy

\section{Publish your work in this journal}

OncoTargets and Therapy is an international, peer-reviewed, open access journal focusing on the pathological basis of all cancers, potential targets for therapy and treatment protocols employed to improve the management of cancer patients. The journal also focuses on the impact of management programs and new therapeutic agents and protocols on

\section{Dovepress}

patient perspectives such as quality of life, adherence and satisfaction The manuscript management system is completely online and includes a very quick and fair peer-review system, which is all easy to use. Visit http://www.dovepress.com/testimonials.php to read real quotes from published authors. 